\title{
La categoría Estado en el curriculum de la Escuela de Trabajo Social ${ }^{1}$ The State category in the curriculum of the School of Social Work
}

\author{
Yessenia Fallas Jiménez \\ Escuela de Trabajo Social, UCR, San José, Costa Rica \\ yessenia.fallasjimenez@ucr.ac.cr \\ https://orcid.org/0000-0003-4345-5657
}

\begin{abstract}
Resumen
Introducción: El proyecto de investigación Fundamentos históricos de la génesis del Estado moderno y su relación con la producción capitalista realizó entre otras actividades un análisis de la forma en la que se aborda en la Escuela de Trabajo Social la discusión sobre el Estado y la política social a partir de los cursos Teoría del Estado y Política Social. Objetivo: el primer objetivo del proyecto establece: «Identificar los principales fundamentos teóricos dentro del campo marxista que aportan elementos para la comprensión del Estado moderno», derivado del estudio de las fuentes teóricas dentro del campo marxista, del cual se realizó un análisis del contenido y de los aspectos metodológicos contenidos en los cursos para el abordaje de estas categorías.

Método: la investigación centra su atención en elementos técnicos referidos a la investigación bibliográfica y documental. Para el abordaje de este análisis se estudió el programa de ambos cursos identificando tendencias en la estrategia pedagógica y en la perspectiva teórica de los contenidos de ambos programas.

Resultados: se identificó el predominio de una tendencia fragmentaria en el abordaje de las categorías Estado y política social, aprehendiendo los elementos históricos como planos de fondo historeográficos para el análisis de los objetos y no como elementos que constituyen la naturaleza social de los mismos.

Conclusiones: si bien es posible identificar en los contenidos de los cursos algunos asuntos referidos al proceso histórico, los aspectos metodológicos del abordaje fragmentan la comprensión de dicho proceso, esto al privilegiar un análisis tecnicista y administrativista del Estado y la política social.
\end{abstract}

Palabras clave: Capitalismo, Política, Producción, Pedagogía, Formación.

1 Este texto fue presentado como ponencia en la Jornada de Investigación de la Escuela de Trabajo Social 2021 denominada Tendencias Contemporáneas de la Investigación en Trabajo social. 
DOI 10.15517/ rr.v101i2.49273

\begin{abstract}
Introduction: The research project Historical foundations of the genesis of the modern State and its relationship with capitalist production conducted among other activities an analysis of the way in which the discussion on the State and social policy is approached in the School of Social Work from the courses State Theory and Social Policy.

Objective: The first objective of the project states: "Identify the main theoretical foundations within the Marxist field that provide elements for the understanding of the modern State", derived from the study of the theoretical sources within the Marxist field, an analysis of the content and methodological aspects contained in the courses for the approach of these categories was carried out.

Method: The research focuses its attention on technical elements referred to bibliographic and documentary research. For the approach of this analysis, the program of both courses was studied, identifying tendencies in the pedagogical strategy and in the theoretical perspective of the contents of both programs.

Results: The predominance of a fragmentary tendency was identified in the approach to the categories State and Social policy, apprehending the historical elements as historiographic background blueprints for the analysis of the objects and not, as elements that constitute the social nature of the objects.
\end{abstract}

Conclusions: Although it is possible to identify in the contents of the courses some issues referred to the historical process, the methodological aspects of the approach fragment the understanding of this process by privileging a technical and administrativist analysis of the State and social policy.

Keywords: Capitalism, Politics, Production, Pedagogy, Training.

\title{
Introducción
}

El presente documento es parte de los resultados del proyecto de investigación supracitado, desarrollado durante los años 2018-2021. Como parte de las indagaciones investigativas surgió la necesidad de identificar asuntos metodológicos referidos a la comprensión de la categoría Estado, así como a la forma en la que las discusiones sobre dicha categoría son tratadas en la formación profesional, particularmente en una profesión cuya reproducción está orgánicamente articulada a los desarrollos históricos del Estado moderno.

El análisis de los elementos presentados en este texto se limita a los contenidos de dos cursos que son centrales en la discusión sobre Estado durante la formación de profesionales en la Escuela de Trabajo Social de la Universidad de Costa Rica, a saber, el curso TS-4016 y TS-0046 Teoría del Estado y Política social I y II, respectivamente (Escuela de Trabajo Social, ETSoc 20019a). 
Como resultado de la reforma parcial al plan de estudios realizada en el año 2004, estos cursos fueron ubicados en el IV nivel de la carrera (ETSoc 20019a), siendo posteriormente reubicados en el tercer nivel a partir de una nueva reforma parcial en el año 2016 con el argumento de que en este nivel se imparte el primer curso de práctica, por lo que sería fundamental acompañar este ejercicio académico de acercamiento a la intervención profesional con una discusión sobre las categorías Estado y política social.

En esta segunda reforma parcial cambia la localización de los cursos, pero no su contenido, que con pequeñas variaciones se ha mantenido estable a lo largo de los años. No interesa en el presente texto hacer un análisis comparativo de los programas de los cursos desde el año 2004, sino más bien analizar los contenidos y la propuesta de abordaje de las categorías supracitadas que se ofrece actualmente en dichos programas.

Para tal efecto, se localizó el objeto específico de análisis en el año 2019, es decir, la discusión contenida en este texto refiere a los programas de ambos cursos aprobados por la Comisión Docencia de la Escuela de Trabajo Social en el año 2019. Es importante aclarar que como parte de las adecuaciones realizadas en el marco de la pandemia por COVID-19, ambos programas sufrieron cambios, específicamente referidos a la cantidad de contenidos desarrollados, esto en acatamiento a la resolución VD-11502-2020 que establece un máximo de horas de clase a través de la plataforma virtual METICS para cada curso (UCR 2020) y de la cantidad de productos evaluables.

\section{¿Por qué la discusión sobre el Estado y la política social es fundamental para la profesión del trabajo social?}

Debe aclararse que la naturaleza de una profesión cuyo origen se encuentra articulado a las formas en las que el Estado ejerce su función de cara al proceso de acumulación de capital (Villela Iamamoto 1995) hace que la comprensión de los desarrollos históricos que dan origen tanto al Estado como a la política social sea medular en la formación de profesional.

La reproducción del trabajo social en cuanto profesión que surge como expresión concreta del desarrollo capitalista y de la necesaria división social del trabajo ${ }^{2}$ incorpora como mediación constitutiva de su existencia el desarrollo de políticas públicas y sociales, las cuales se desdoblan como realizaciones concretas de la función social del Estado moderno en el marco del proceso de reproducción ampliada del capital.

La segunda mitad del siglo XX fue un escenario privilegiado para identificar el vínculo constitutivo de la profesión con el Estado y como consecuencia con el proceso de reproducción del capital, pues la función social del Estado moderno en ese periodo amplió vigorosamente su rango de expresiones como parte de las respuestas a la crisis de 1929. La

2 Pero también por la división socio técnica, pues es justamente la necesidad de la persona profesional que administre la ejecución de la política social lo que determina su existencia (Villetla Iamamoto 1992). 
ampliación del Estado como coadyuvante en la amplitud de los mercados de trabajo (por ejemplo, con el aumento en la creación de instituciones estatales y como consecuencia de la contratación de una masa de personal trabajador público) y de la política social como formas de intensificación de la extracción de plusvalía relativa crearon condiciones para la plena reproducción de una profesión cuya función social se concreta en la planificación y ejecución de las políticas sociales.

Es verdad que los países centrales tuvieron condiciones particulares de crecimiento dada su naturaleza industrial, pero también la periferia vivió expresiones de ampliación del escenario público que contribuyeron a la formación de un colectivo de trabajadores con un estatuto de corte primero técnico y luego profesional.

Llegada la década de los setenta y con ello el inicio de una crisis de naturaleza estructural, fue posible observar el desarrollo de tentativas de superación de la crisis que, como era de esperarse, implicaron la actuación del Estado y con ello la «reforma» de su estructura, ahora interpretada como una estructura hipertrofiada que debía someterse a los «saludables» principios de la apertura comercial y el achicamiento. La llegada de las políticas neoliberales, como intento de superación de la crisis, impactó entonces la «enorme» estructura pública creada y legitimada por su función social en las décadas anteriores, implementado ahora, junto con la reducción y la apertura comercial, políticas sociales de carácter focalizado.

En ese proceso de transformaciones el trabajo social ha sufrido consecuencias, tanto en las formas históricas a través de las cuales se legitima como en las formas en las que se reproduce cotidianamente. Son justamente los vínculos que esas transformaciones tienen con la profesión (por su carácter y origen) que colocan la necesidad imperiosa de entender cuatro elementos centrales en la existencia y reproducción de la profesión:

1. La constitución histórica de ese espacio de reproducción profesional (que, aunque no es el único, es el predominante), es decir, el Estado moderno.

2. Las existencias de formulaciones teóricas y políticas que a lo largo de su existencia se tornaron como las bases explicativas que, en mayor o menor medida, lograron una aprehensión genuina de su constitución histórica.

3. La política social como forma de realización de la función del Estado moderno y su relación con el proceso de acumulación de capital.

4. Las mediaciones que articulan la relación orgánica entre Estado, política social y trabajo social.

Este conjunto de elementos reafirma la necesidad de que, a lo largo de la formación la población estudiantil comprenda los objetos y los procesos de trabajo relativos a la intervención profesional, como elementos que necesariamente se constituyen a partir de la mediación del proceso de acumulación de capital y del desarrollo del Estado en el marco de dicho proceso, entre otras mediaciones. 
Aclarado lo anterior corresponde ahora analizar la forma en la que esta categoría se aborda en los dos cursos mencionados.

La estructura de los cursos de la Escuela de Trabajo Social está organizada a partir de objetivos de conocimiento, de habilidades y de actitudes. En este análisis la atención estará centrada en los objetivos de conocimiento, esto debido a que interesa analizar la forma en la que se aborda, a partir de los contenidos y unidades temáticas, la discusión sobre la categoría Estado moderno (ETSoc 2019b). En términos de los objetivos del curso TS4016 Teoría del Estado y Política Social I, se plantea lo siguiente):

1. Identificar y caracterizar los enfoques teóricos más importantes acerca del Estado, la sociedad y las políticas públicas, así como brindar algunos elementos de economía política relacionados con el tema.

2. Analizar las racionalidades que configuran el Estado y las políticas públicas.

3. Relacionar los problemas teóricos y prácticos sobre la naturaleza del Estado y su relación con la práctica profesional del trabajo social en el marco de las políticas sociales.

4. Identificar las principales concepciones de reforma del Estado a la luz de algunas experiencias particulares en América Latina y en los países capitalistas avanzados y sus implicaciones en la trayectoria de la política social.

5. Conocer la instrumentalidad técnica y política inherente a las políticas sociales y su influencia en la práctica profesional del trabajo social.

6. Puede notarse en la organización de estos objetivos que el primer curso coloca como finalidad una discusión sobre las perspectivas teóricas de abordaje de la categoría Estado, las transformaciones del Estado a partir de la década de 1980 según lo que se denomina «reforma del Estado» y la vinculación con el Trabajo Social y su intervención profesional.

Llama la atención el segundo objetivo que plantea el análisis de lo que se denomina «racionalidades que configuran el Estado», lo que puede entenderse en vinculación con procesos histórico más amplios, más específicamente el proceso de acumulación de capital (aunque no se nomina específicamente así).

Como puede verse, a pesar de que el curso propone un acercamiento a debates teóricos y comprensiones filosóficas sobre el Estado (como veremos en los contenidos más adelante) no existe una estrategia que encamine la comprensión del surgimiento del Estado moderno a partir de las determinaciones histórico-materiales que lo configuran como estructura política en la sociedad burguesa.

De acuerdo con la ETSoc (2019b), en el caso del curso TS 0046 Teoría de Estado y Política Social II, observamos los siguientes objetivos:

1. Conocer diferentes perspectivas teóricas acerca de las políticas sociales.

2. Identificar los escenarios y contextos en los que se ha enmarcado el desarrollo de las políticas sociales en general y en particular en Costa Rica.

3. Caracterizar de manera práctica las políticas sociales en Costa Rica y estudiar el papel político que asumen en la construcción del orden social. 
4. Determinar cómo se configura, se construye y deconstruye la política social, esto a partir del juego de intereses presentes en la dinámica político-social.

5. Conocer las principales propuestas de análisis de las políticas sociales utilizadas en la actualidad.

Se discutirán más adelante los contenidos de ambos cursos, pero nótese desde el planteamiento de los objetivos un asunto: en el segundo curso parece privilegiarse una comprensión que toma como punto de partida el contexto del objeto de estudio, que ya en este caso no se enfoca en el Estado, sino en la política social, lo que no es posible observar en el primer curso, donde la categoría Estado parece estar separada de los procesos históricos más amplios.

Para ver estos elementos con detalle es necesario conocer los contenidos de los cursos, en ambos casos dichos contenidos se organizan en unidades temáticas que a su vez contienen un conjunto de tópicos a ser desarrollados, tal y como se observa en la Tabla 1:

Tabla 1. Contenidos temáticos del curso TS4016: Teoría del Estado y Política Social I, 2019

Unidad

I UNIDAD:

Estado y sociedad

II UNIDAD: política social,
Contenidos

I.1 Abordajes teóricos sobre «Estado» y «Sociedad», según personas autoras clásicas y contemporáneas.

- Contractualistas (Hobbes, Locke, Rousseau)

- No Marxistas (Hegel, Durkheim, Weber, Parsons)

- Marxistas (Marx y Engels, Lenin, Gramsci, Poulantzas)

- Neomarxistas (Offe, Jessop, Hirsh)

I.2 Naturaleza del Estado

- Elementos que le caracterizan como fenómeno histórico y relacional

- Diferencias entre Estado/ Gobierno / Nación

- Sistemas políticos, administración pública e institucionalidad

- Nueva gerencia pública y acción estatal

- El enfoque de derechos humanos, interculturalidad y políticas públicas

I.3 Sociedad civil, ciudadanía, y participación social

Espacio público y ciudadanía

Trilogía: Estado/ Mercado / Sociedad civil

Nuevos movimientos sociales y participación social (tensiones y contradicciones)

II.1 Concepciones tradicionales y críticas de la política 
reforma del Estado y re- social. configuración de lo político II.2 Trayectoria de la política Social en América Latina y Costa

- Políticas Sociales de la Ley de Los Pobres.

Rica

- Políticas Sociales del Estado de Bienestar

- Políticas Sociales del Estado Neoliberal

III UNIDAD: Estado, II.3 «Reforma del Estado» y políticas sociales: América política social y ejercicio Latina y Costa Rica

profesional

- El ajuste de I Generación: el Consenso de Washington en América Latina

- El ajuste de segunda generación y la reforma institucional.

- Las implicaciones de las reformas del Estado en las políticas sociales

- La reorientación de la reforma de la política social: entre el universalismo, la focalización y la condicionalidad

- Desafíos y retos actuales de las políticas sociales

III.1 Políticas públicas y política social

- El aporte de estudio de las políticas públicas a la comprensión del Estado y las políticas sociales

- El diseño y análisis de política pública

III. 2 Condición actual de la política social en Costa Rica

- Tendencias contemporáneas, determinantes, características.

- Límites, posibilidades y alternativas.

III.3 Ejercicio profesional en el marco de la política social contemporánea.

- Desigualdades, nueva cuestión social, institucionalidad y compromiso ético

Fuente: elaboración de la autora a partir de ETSoc, Universidad de Costa Rica. $2019 \mathrm{~b}$. Programa de los cursos TS-4016 y TS-0046. I. San José, Costa Rica: ETSoc-UCRETSocUCR.

Es importante resaltar que en la primera unidad de este curso se propone una comprensión de dos categorías (Estado y sociedad) y a pesar de que los contenidos de los puntos uno y dos (parcialmente) muestran una aproximación a las mismas, nótese que a partir de la mitad del punto dos hay una transición hacia un contenido que ya no pertenece a la comprensión del Estado y la sociedad como elementos que deben ser aprehendidos a 
partir de procesos históricos más amplios, puede pensarse, por ejemplo, la Revolución industrial, la Revolución francesa, y el modo de producción capitalista.

Específicamente en el punto dos de la segunda unidad que se denomina «Naturaleza del Estado», el curso coloca elementos de carácter histórico así como otros que parecen ser mediaciones más contemporáneas referidas a la formas administrativas y de gestión burocrática del aparato público: «sistemas políticos, administración Pública e institucionalidad, nueva gerencia pública y acción estatal» para posteriormente terminar con una discusión sobre «enfoques» normativos y conceptuales relativos a las políticas públicas (el enfoque de derechos humanos, interculturalidad y políticas públicas).

Si bien el punto uno de la primera unidad centra su atención en la compresión de diferentes perspectivas teóricas para el análisis del Estado, el punto dos de esta misma unidad transita por elementos de carácter histórico, teórico e incluso técnicoadministrativos sobre el Estado moderno. Es interesante observar que, siempre en la primera unidad, en el punto tres se introduce la discusión de lo que se denomina «sociedad civil», que parece referirse al espacio que no responde ni a la esfera del Estado, ni a la del mercado, con la intención de abordar el papel de los sujetos sociales como elemento correlativo a la reproducción del Estado moderno.

En síntesis, en la primera unidad del curso vemos tres grandes ejes temáticos: perspectivas teóricas sobre la concepción de Estado, elementos de carácter administrativo relativos a la reproducción del Estado y, finalmente, una discusión sobre lo que se denomina sociedad civil, como elemento diferenciador tanto del Estado como del mercado.

Al pasar a la segunda unidad de este curso, denominada «Política social, reforma del Estado y re-configuración de lo político en América Latina y Costa Rica», observamos un elemento interesante: se retoman aspectos propios del proceso histórico, a pesar de ello la discusión está angulada a partir de dos criterios, el primero es que el objeto ya no es el Estado, sino la política social (y las transformaciones de la misma a partir de la llamada reforma del Estado) y el segundo refiere al hecho de que la presencia de estos aspectos del proceso histórico son aprehendidos cronológicamente y no a partir de la base material que los sustenta, es decir, como momentos específicos de un proceso histórico que no inicia en el siglo XX, sino mucho antes. Esto se expone como antesala de la discusión final de esta unidad, a saber, el desarrollo del neoliberalismo y su impacto en la política social.

En síntesis, vemos en esta segunda unidad tres ejes temáticos: discusiones teóricas (ahora no de Estado, sino de política social), un recorrido cronológico alrededor de las formas clásicas en las que se desarrolla la política social y, finalmente, una discusión acerca del neoliberalismo y sus derivaciones en la reforma del Estado y la política social.

Dos elementos son fundamentales en relación con estas dos primeras unidades, ambos pertenecientes a la esfera del método. En primer lugar, la discusión fragmentada de las dos categóricas centrales en el curso, Estado y política social; en segundo lugar, la 
incorporación de elementos de carácter histórico que, en principio, son reducidos a su aspecto cronológico. Al no aprehender el proceso histórico más amplio referido a la acumulación de capital, se pasa por alto la relación orgánica entre este proceso y la naturaleza del Estado moderno (y como consecuencia la política social). Esto, aunado al hecho de que las dos primeras unidades transitan por cuestiones teóricas, históricas y administrativas de la estructura del Estado, podría generar la impresión de que esos tres asuntos tienen el mismo peso en la constitución y la reproducción del Estado moderno.

Ya en la tercera y última unidad se observa que la centralidad temática está colocada de lleno en la política social, pero el acercamiento propuesto no se hace tampoco desde una óptica histórica, puede decirse que esta unidad transita por tres ejes: la política pública y social (con una perspectiva administrativista de la política), las actuales condiciones de la política social en Costa Rica y la relación entre la política y el trabajo social.

Ahora bien, al revisar los contenidos del curso TS 0046: Teoría del Estado y Política Social II, se observa, en primer lugar, que a pesar de que ambos cursos se constituyen como una gran área temática de formación (Teoría del Estado y política social) organizada en dos ciclos lectivos, el segundo curso último inicia retomando algunos elementos generales de la teoría del Estado (que fue parte del programa del curso anterior), según se observa en la tabla 2.

Tabla 2. Contenidos temáticos del curso TS 0046: Teoría del Estado y Política Social II, 2019

Unidades Temáticas: Perspectivas teórico-metodológicas para el análisis de la política pública/social

I Unidad

1. Introducción:

Relaciones Estado, cuestión social, movimientos sociales y política social

2. Enfoques teóricos para el estudio de las políticas sociales:

- Teoría de la Ciudadanía

- Concepción instrumentalista

- Enfoque estatista

- Enfoque politicista

- La perspectiva marxista

3. Evolución del sistema capitalista.

4. Siglo XX proceso de acumulación de capital, Estado y transformaciones en la Política Social

5. Propuestas de reestructuración de las políticas públicas/ sociales en el contexto neoliberal. 
II Unidad

III Unidad
1. Finalidad y ciclo de la política pública: surgimientoorigen, formulación, gestión, implementación, seguimiento y evaluación.

2. Componentes fundamentales en el proceso de formación de la política pública: capital, necesidades y demandas, conflicto social, conformación de agendas, actores sociales, procesos de negociación, respuestas estatales y no estatales.

3. Reformas económicas y política social costarricense

4. Política pública/social y ejercicio profesional: contextualización desde los espacios locales, o bien sectores de práctica académica.

\section{Sistema Nacional de Planificación}

1. Tipos de análisis de la política pública: análisis descriptivos, análisis prescriptivos y análisis desde una perspectiva marxista.

2. Propuestas para el análisis de la política pública: modelo del ciclo político, análisis de coyuntura, redes de políticas públicas, teoría de las coaliciones, teoría neoinstitucional, regímenes de bienestar, perspectivas neomarxistas.

Fuente: elaboración de la autora a partir de ETSoc, Universidad de Costa Rica. 2019b. Programa de los cursos TS-4016 y TS-0046. I. San José, Costa Rica: ETSoc-UCRETSocUCR.

Un primer elemento pedagógico que salta a la vista es el hecho de que las unidades temáticas de este curso no están claramente tituladas y el curso parece girar alrededor de un tema específico: «Perspectivas teórico-metodológicas para el análisis de la política pública/social».

Si se observa la primera unidad, llama la atención el hecho de que inicie con una discusión particularizada (y también abordada en el curso anterior) sobre Estado, política social y movimiento sociales, (adicionalmente se coloca la llamada cuestión social) para pasar posteriormente a un tema («evolución del sistema capitalista» y «siglo XX proceso de acumulación de capital, Estado y transformaciones en la Política Social») cuya complejidad amerita no solamente que sea colocado al inicio de este curso, sino incluso que sea parte del curso anterior, pues por la densidad teórica y el ejercicio metodológico que demanda hace necesario tener una base de conocimiento sobre procesos históricos más amplios que no fueron proporcionados en el curso anterior. Ya en el segundo punto de esta unidad se 
reproduce de alguna manera la escisión entre el proceso histórico de acumulación, el Estado y la política social, esto cuando se abordan los denominados «enfoques teóricos» para el análisis de las políticas.

Esta primera unidad del curso claramente cuenta con tres ejes temáticos, uno que es colocado como introducción y se refiere a la relación entre Estado-política social y movimientos sociales, un segundo eje que aborda aspectos teóricos referidos al análisis de políticas desde diferentes perspectivas técnicas y, finalmente, un eje que se centra en aspectos de carácter histórico más amplio, particularmente relacionados al proceso de acumulación de capital. Esta es la primera vez que este contenido se explicita en ambos cursos y, en ese sentido, vale la pena preguntarse sobre la pertinencia de que esta discusión esté colocada en el segundo curso y no en el primero.

Ya en la segunda unidad se observa más claramente la intención pedagógica de este segundo curso, a saber: el análisis de las políticas públicas y sociales. Un elemento importante es que a pesar de que hay un enfoque tecnicista tanto de la formulación de políticas como de la comprensión del análisis que de la misma debe hacerse, se mencionan algunos aspectos del proceso histórico que deben contemplarse, por ejemplo: capital, conflicto social, reformas económicas. A pesar de ello, al organizar la unidad en ejes temáticos, se observa una dispersión de temas contenidos en la misma: aspectos burocráticos de la política (lo que en la unidad se llama ciclo de la política), reformas económicas y políticas social en Costa Rica, políticas sociales y trabajo profesional. Finalmente, se haya un tema que se separa de la lógica temática de la unidad, el «Sistema Nacional de planificación».

En la última unidad se puede identificar un solo eje temático: el análisis de la política social, este eje se divide en dos elementos: lo que en el programa se denomina «tipos de análisis» y «propuestas de análisis».

Un asunto que llama la atención en los dos programas analizados es que, a pesar de que hay una presencia de elementos de carácter histórico, los mismos no aparecen como perspectiva metodológica fundamental para la comprensión de las categorías más importantes en ambos cursos (Estado y política social). Priva el abordaje de la historia desde una óptica cronológica, no necesariamente desde una premisa metodológica, sin la cual el contenido de ambas categorías no se comprende en su movimiento contradictorio como parte de una totalidad social más amplia.

Si bien los contenidos ofrecidos por ambos cursos son en su mayoría importantes para el análisis de las categorías centrales, los mismos están dispersos a lo largo de ambos programas, en algunos casos se reiteran y no hay una perspectiva clara de la relación procesal en el tratamiento de las categorías.

Ahora bien, sobre la perspectiva teórica que subyace en los programas, puede decirse que no hay una línea de abordaje específica de ambas categorías, más bien 
predomina una tendencia a «repasar» varias perspectivas de comprensión tanto de Estado y política social como de las formas en las que el análisis de las mismas debe ser realizado, sin privilegiar uno de ellos como perspectiva hegemónica de los cursos, lo que sí es posible observar en otros cursos de la malla curricular de la Escuela (por ejemplo en el curso TS0017 Teoría y Método del trabajo social II). Esto deja a la voluntad del personal docente el desarrollo de la perspectiva que va a privilegiar en el abordaje de los temas, lo que, si bien no riñe con la libertad de cátedra, no permite entender la direccionalidad que la Escuela pretende dar en el abordaje de ambas categorías.

En ese sentido, lo delicado de este asunto es el hecho de que la falta de una línea de desarrollo teórico específica desde el programa, termina propiciando un abordaje eclecticista que, en término generales, tiende a privilegiar una comprensión administrativista, tecnicista y politicista, en vez de un abordaje histórico.

Por otro lado, en términos de la cuestión metodológica en el abordaje de las ambas categorías, tal y como se mencionó anteriormente, si bien se incorporan algunos elementos teóricos y filosóficos más amplios referidos al Estado moderno, no hay una perspectiva histórica en la aprehensión de esta categoría, sino más bien historiográfica. En este sentido, el desarrollo del Estado moderno tiende a abordarse más como un asunto conceptual que histórico. Así, en la comprensión del Estado los procesos históricos más amplios, como por ejemplo la acumulación de capital, parecen ser tratados como un «marco de referencia» junto con otros marcos y no como una propuesta pedagógica (y por lo tanto teórico y política) del curso.

Ya en términos generales, se observa una fragmentación entre los asuntos de carácter teórico e histórico, como si ambos no estuvieran articulados, como si lo teórico no fuera el resultado de la captación racional del movimiento de la realidad, de manera que, por ejemplo, en ambos cursos, el análisis del Estado y la política social desde el marxismo son tratados como elementos temáticos, pero no se propicia la habilitación del estudiantado para hacer ese tipo de análisis.

Al no ser el proceso histórico la base para la comprensión del Estado, el análisis de las políticas sociales tiende a deshistorizarse, privilegiando un análisis conceptual y administrativista de la política, lo que implica que no se aprehendan las transformaciones de las cuales la misma es objeto, teniendo como punto de partida el proceso histórico más amplio referido específicamente a la acumulación de capital.

Es posible observar que el curso TS 0046 promueve el ejercicio de análisis de la política social desde una perspectiva más técnica que histórica, se privilegia un abordaje de enfoques, perspectivas y modelos de análisis en vez de «preparar» a las y los estudiantes metodológicamente para entender la naturaleza del Estado moderno y la función social que cumple en el proceso de acumulación de capital, lo cual hace énfasis con ello en elementos técnicos, administrativos y políticos más que en los procesos históricos que los determinan. 
DOI 10.15517/ rr.v101i2.49273

Lo anterior no quiere decir que el análisis de la particularidad de las políticas sociales no sea importante, por el contrario, es justamente su importancia la que demanda una comprensión de las mismas como síntesis de otros procesos que superan la existencia individual de una normativa, una ley, un programa o una política propiamente dicha. Tampoco quiere decir que la formación acerca de recursos operativos para el análisis de políticas no deba ser materia del curso, si no que los mismos no pueden limitarse a los aspectos más aparentes de las políticas como objetos de estudio.

\section{A modo de conclusión}

La debilidad en la comprensión del Estado moderno articulado a desarrollos históricos más amplios (acumulación, crisis del capital, etc,) podría convertirse en un obstáculo para entender dos asuntos. En primer lugar, de qué manera la política social contribuye de diferentes formas y a lo largo de la historia al proceso de acumulación de capital, véase por ejemplo la producción de plusvalía relativa, particularmente en los países centrales durante los gloriosos años del keynesiamos o la transición a las políticas de transferencias monetarias condicionadas en el periodo de la crisis estructural del capital, solamente para citar dos ejemplos. En segundo lugar, la contradicción sobre la cual se reproduce el trabajo social como categoría profesional, identificando los límites y alcances de la política social, así como su impacto en la reproducción de una profesión cuya naturaleza está imbricada en la forma en la que se constituyó el Estado moderno como estructura política en la sociedad burguesa.

Estos dos elementos deben ser analizados por la Escuela de Trabajo Social, pues la urgencia de una respuesta acorde a la carga del tiempo histórico nos coloca desafíos importantes para la formación profesional.

\section{Referencias}

Fallas, Yessenia. 2018. «Fundamentos históricos de la génesis del Estado moderno y su relación con la producción capitalista». Proyecto de Investigación. San José, Universidad de Costa Rica.

TSoc-UCR (Escuela de Trabajo social, Universidad de Costa Rica). 2019a. Plan de estudios de la carrera de Trabajo Social. San José, Costa Rica: ETSoc-UCR 2019b. Programa de los cursos TS-4016 y TS-0046. I. San José, Costa Rica: ETSocUCRETSOc-UCR.

Universidad de Costa Rica. 2020. Resolución VD-11502-2020. Lineamientos académicos y administrativos para la docencia con componente virtual. San José, Universidad de Costa Rica.

Villela Iamamoto, Marilda. 1992. Servicio social y división del trabajo. São Paulo, Brasil: Cortez Editora. 